\title{
TESTES DE VIGOR NA AVALIAÇÃO DA QUALIDADE FISIOLÓGICA DE SEMENTES Erythrina velutina Willd. (FABACEAE - PAPILIONOIDEAE)
}

\author{
Vigor tests in the evaluation of physiological quality of \\ Erythrina velutina Willd. (FABACEAE- PAPILIONOIDEAE) seeds \\ Roberta Sales Guedes ${ }^{1}$, Edna Ursulino Alves ${ }^{2}$, Edilma Pereira Gonçalves ${ }^{3}$, \\ Severino do Ramo Nascimento dos Santos ${ }^{4}$, Cosmo Rufino de Lima ${ }^{5}$
}

\begin{abstract}
RESUMO
A aplicação de testes de vigor de sementes tem sido fundamental para determinação da qualidade fisiológica de forma rápida e eficaz, destacando-se, os testes baseados no desempenho das plântulas como uma das opções disponíveis. Conduziu-se este trabalho, com o objetivo de avaliar a eficiência de testes de vigor para determinação da qualidade fisiológica de sementes de Erythrina velutina. Determinou-se em cinco lotes de sementes de E. velutina o teor de água e o peso de mil sementes e realizou-se os testes de germinação, emergência em campo, índice de velocidade de germinação e de emergência, comprimento e massa seca de plântulas do laboratório e campo. Os testes de campo (emergência de plântulas, índice de velocidade de emergência de plântulas e massa seca da parte aérea das plântulas) são eficientes na separação dos lotes de sementes de E. velutina em níveis de vigor.
\end{abstract}

Termos para indexação: Mulungu, germinação, vigor, emergência, Erythrina velutina.

\section{ABSTRACT}

The application of vigor tests of seeds has been fundamental as a fast and efficient form of determination of physiological seed quality, highlighting the tests based on the performance of seedlings as one of the available options. The objective of this study was to evaluate the efficiency of different vigor tests for the determination of the physiologic quality of Erythrina velutina seeds. In five lots of E. velutina seeds, water content and seed weight were determined. Tests for germination were performed for field seedling emergence, speed germination and emergence index, and length and dry matter weight of seedlings from both the laboratory and from the field. Field tests (seedling emergence, speed emergence index, and dry matter weight of the aerial part of seedlings) are efficient in the separation of the seed lots of E. velutina in vigor levels.

Index terms: Mulungu, germination, vigor, emergence, Erythrina velutina.

(Recebido em 29 de maio de 2008 e aprovado em 2 de outubro de 2008)

\section{INTRODUÇÃO}

A qualidade das sementes pode ser determinada por vários testes, como o teste de germinação, o qual é conduzido sob condições controladas, sendo muito útil para avaliar a capacidade de germinação, no entanto, não subsidia informações sobre o vigor das sementes (Bewley \& Black, 1994).

Os testes de vigor permitem identificar os lotes com maior ou menor probabilidade de apresentar melhor desempenho no campo ou durante o armazenamento. São instrumentos importantes como adjuntos ao teste de germinação na pesquisa sobre qualidade de sementes
(Hampton \& Coolbear, 1990), pois uma das principais exigências para a avaliação do vigor de sementes refere-se à obtenção de resultados confiáveis em um período de tempo relativamente curto, permitindo agilizar decisões quanto ao manejo de lotes, durante as etapas de póscolheita das sementes.

O vigor das sementes é função de um conjunto de características que determinam o potencial para emergência rápida e uniforme de plântulas normais, sob ampla diversidade de condições ambientais (AOSA, 1983). Desse modo, a utilização de um único teste de vigor (fisiológico, bioquímico, ou de resistência a condições de estresse)

'Bióloga, Mestre em Agronomia, Produção e Tecnologia de Sementes - Fitotecnia - Rua Tristão Granjeiro, 579 B - Centro - 58397-000 - Areia, PB roberta_biologa09@yahoo.com.br

${ }^{2}$ Agrônoma, Doutora em Agronomia, Produção e Tecnologia de Sementes - Fitotecnia - Centro de Ciências Agrárias da Universidade Federal da Paraíba - CCA UFPB - Vila dos Professores - Cx. P. 02 - Centro - 58397-000 - Areia, PB - ednaursulino@cca.ufpb.br

${ }^{3}$ Agrônoma, Doutora em Agronomia, Produção e Tecnologia de Sementes - Fitotecnia - Universidade Federal Rural de Pernambuco - Unidade Acadêmica de Garanhus - Avenida Bom Pastor, s/n - Boa Vista - 55296-901 - Garanhus, PE - edilmapg@hotmail.com ${ }^{4}$ Agrônomo - Fitotecnia, Centro de Ciências Agrárias da Universidade Federal da Paraíba/CCA/UFPB - Rua Semeão Leal, 269 - Centro - 58397-000 Areia, PB - ninoagro@hotmail.com

${ }^{5}$ Agrônomo - Fitotecnia - Centro de Ciências Agrárias da Universidade Federal da Paraíba/CCA/UFPB - Rua Semeão Leal, 269 - Centro - 58397-000 Areia, PB - cosmoagr@hotmail.com 
pode gerar informações incompletas (Hampton \& Coolbear, 1990). Marcos Filho (1999) recomenda na avaliação do vigor de sementes, a utilização da combinação dos resultados de diferentes testes, levando-se em consideração a finalidade do uso dos resultados e suas limitações.

Conforme Nakagawa (1999), a primeira contagem do teste de germinação pode ser utilizada como um teste de vigor, uma vez que a velocidade de germinação é reduzida com o avanço da deterioração da semente. Assim, amostras que apresentam maiores valores de germinação na primeira contagem podem ser consideradas mais vigorosas. Tratase de um teste simples e de fácil execução, mas que geralmente apresenta baixa sensibilidade, detectando pequenas diferenças de vigor entre os lotes. Um outro teste que é preciso na comparação do vigor entre lotes é a emissão da raiz primária (Bewley \& Black, 1994).

O teste de primeira contagem é eficiente na separação de lotes de sementes Calendula officinalis L. em níveis de vigor (Silveira et al., 2002), e também em sementes de Ricinus communis L. (Fonseca et al., 2004), Cucumis melo L. (Bhering et al., 2004), feijão - macassar (Torres \& Bringel, 2005) Beta vulgaris L. (Silva \& Vieira, 2006) e Erythrina velutina Willd. (Guedes et al., 2008).

São consideradas mais vigorosas as amostras de sementes que originam plântulas com maiores valores de comprimento da parte aérea e massa verde ou seca, num mesmo período de tempo (AOSA, 1983), sendo esta característica também utilizada como um dos testes de vigor. Isto ocorre em função das sementes conterem maior suprimento de reservas nos tecidos de armazenamento, desse modo, maior massa e maior competência de transformação destas reservas em substâncias que podem ser assimiladas pelo eixo embrionário (Taiz \& Geizer, 2006).

Em razão do crescente interesse pelas espécies florestais nativas, tornam-se importantes tanto a caracterização da qualidade de suas sementes, por meio de testes de germinação e vigor, como a obtenção de informações sobre a eficiência e rapidez de diferentes testes de vigor para se estimar a qualidade das sementes dessas espécies. O mulungu (Erythrina velutina) por apresentar considerável importância econômica (Kurihara et al., 2005) e na medicina popular (Lorenzi, 2002) merece atenção especial quanto à avaliação do seu potencial fisiológico, para tanto, estudos dirigidos à determinação do vigor devem ser realizados por não existirem informações sobre a espécie. Neste estudo, objetivou-se comparar a eficiência de testes de vigor, visando a avaliar a qualidade fisiológica de lotes de sementes de Erythrina velutina Willd. (Fabaceae - Papilionoideae)

\section{MATERIALE MÉTODOS}

A pesquisa foi conduzida no Laboratório de Análise de Sementes do Centro de Ciências Agrárias da Universidade Federal da Paraíba, em Areia - PB. Foram utilizados cinco lotes de sementes de Erytrhina velutina colhidas manualmente de cinco árvores, em quatro municípios do Estado da Paraíba: Areia $\left(\mathrm{L}_{1}\right.$ e $\left.\mathrm{L}_{2}\right)$, Pocinhos $\left(\mathrm{L}_{3}\right)$, Campina Grande $\left(\mathrm{L}_{4}\right)$ e Remígio $\left(\mathrm{L}_{5}\right)$, avaliando-se as seguintes determinações:

Teor de água - foi realizado conforme Brasil (1992), com utilização de quatro subamostras de 10 sementes colocadas em cápsulas de alumínio em estufa a $105 \pm 3^{\circ} \mathrm{C}$, por 24 horas e os resultados expressos em porcentagem; Peso de 1000 sementes - foi determinado conforme recomendações de Brasil (1992), utilizando-se oito repetições de 100 sementes oriundas da porção semente pura de cada lote, onde cada amostra foi pesada individualmente e o resultado expresso em grama; Teste de germinação - foram utilizadas quatro repetições de 25 sementes, as quais foram previamente escarificadas na região oposta a micrópila, com lixa d'água $\mathrm{n}^{\circ} 80$ e colocadas para germinar em substrato papel "germitest" na forma de rolo, umedecido com água destilada na quantidade equivalente a 3 vezes o peso do papel seco e mantidas em câmeras de germinação (B.O.D.) sob temperatura de $25^{\circ} \mathrm{C}$ e fotoperíodo de 8 horas. Ao final do teste (15 dias), computou-se o número de plântulas normais, segundo o critério proposto por Brasil (1992); O Índice velocidade de germinação (IVG) - foi realizado juntamente com o teste padrão de germinação, sendo as avaliações das plântulas normais realizadas diariamente, à mesma hora, a partir da primeira contagem, cujo cálculo será de acordo com a fórmula proposta por Maguire (1962), onde:

$$
I V G=\frac{G_{1}+G_{2}+\ldots+G_{n}}{N_{1}+N_{2}+\ldots+N_{n}}
$$

em que IVG = índice velocidade de emergência; $G_{1}, G_{2}, \ldots G_{n}$ = número de plântulas normais germinadas a cada dia; $\mathrm{N}_{1}$, $\mathrm{N}_{2}, \ldots \mathrm{N}_{\mathrm{n}}=$ número de dias decorridos da semeadura a primeira e última contagem.

No final do teste de germinação e de emergência em campo foi determinado o comprimento de plântulas. A raiz primária e a parte aérea das plântulas normais de cada repetição foram medidas com o auxílio de uma régua graduada em centímetros, com os resultados expressos em cm/plântula. As mesmas plântulas da avaliação anterior foram colocadas em estufa regulada a $80^{\circ} \mathrm{C}$ por 24 horas para determinação da massa seca de plântulas e, decorrido 
esse período, pesadas em balança analítica com precisão de 0,001 g com resultados expressos em g/plântula (Nakagawa, 1999).

A emergência em campo foi realizada com quatro repetições de 25 sementes de cada lote, as quais foram semeadas em sulcos de 1,0 $\mathrm{m}$ de comprimento e distanciadas $20 \mathrm{~cm}$ entre si a uma profundidade de $1,5 \mathrm{~cm}$. As contagens foram realizadas dos quatro até aos 21 dias após a semeadura e os resultados foram expressos em porcentagem. Conduzido junto com a emergência de plântulas, o índice de velocidade de emergência em campo (IVE) foi realizado, anotando-se, diariamente até o final do teste (21 dias) as plântulas que apresentavam os cotilédones acima da superfície do solo, sendo o IVE calculado empregando-se a fórmula proposta por Maguire (1962).

Os tratamentos consistiram dos cinco lotes de sementes e o delineamento experimental adotado foi inteiramente ao acaso, com quatro repetições. Os dados foram submetidos à análise de variância, sem transformações, e as médias comparadas pelo teste de Scott-Knott a $5 \%$ de probabilidade, quando houve significância no teste F.

\section{RESULTADOS E DISCUSSÃO}

Os valores médios do peso de mil sementes e do teor de água das sementes de Erytrhina velutina encontram-se na Tabela 1, observando-se que houve diferenças significativas entre os lotes. $\mathrm{O}$ lote 1 apresentou sementes significativamente mais pesadas que os demais lotes, sendo que todos os lotes diferiram significativamente entre si, vindo o lote 3 , lote 4 e, por fim, o lote 2 com os menores valores de peso de mil sementes. Possivelmente, esse valor máximo do peso de mil sementes do lote 1 pode estar associado ao elevado teor de água das sementes acima dos demais $(8,17 \%)$.

Segundo Irigon \& Mello (1995), o peso das sementes é relatado como uma das causas morfológicas que podem comprometer o vigor e, assim, influenciar na qualidade fisiológica. Carvalho \& Nakagawa (2000) mencionam que uma semente atinge seu máximo vigor quando apresenta seu máximo peso de massa seca, podendo, após esse ponto, sofrer decréscimo do peso, como resultado de perdas em massa seca pela respiração da semente. Nesse processo há uma diminuição das reservas acumuladas na semente, utilizadas na promoção da germinação e no desenvolvimento inicial de plântula (Carneiro \& Aguiar, 1993). Em sementes de Calendula officinalis L. foi possível a separação de lotes em níveis de vigor pelo peso de mil sementes (Silveira et al., 2002).
O resultado do peso de mil sementes do lote 1 foi compatível com os testes de massa seca de raiz e parte aérea aplicados em campo e em laboratório, uma vez que esse lote continha as sementes mais pesadas, indicando um significativo desempenho na qualidade fisiológica das sementes. Silveira et al. (2002) demonstraram que somente os extremos, ou seja, as classes de maior e menor tamanho exibem qualidade fisiológica inferior em sementes de Calendula officinalis L.

Tabela 1 - Teor de água, e peso de mil sementes de cinco lotes de sementes de Erytrhina velutina.

\begin{tabular}{ccc}
\hline Lotes & $\begin{array}{c}\text { Teor de } \\
\text { água }(\%)\end{array}$ & Peso de mil sementes \\
\hline $\mathrm{L}_{1}$ & $8,17 \mathrm{a}$ & $57,14 \mathrm{a}$ \\
$\mathrm{L}_{2}$ & $7,82 \mathrm{a}$ & $34,97 \mathrm{e}$ \\
$\mathrm{L}_{3}$ & $5,00 \mathrm{c}$ & $40,33 \mathrm{~b}$ \\
$\mathrm{~L}_{4}$ & $5,98 \mathrm{~b}$ & $38,11 \mathrm{c}$ \\
$\mathrm{L}_{5}$ & $6,88 \mathrm{~b}$ & $36,63 \mathrm{~d}$ \\
\hline
\end{tabular}

Médias seguidas de mesma letra na coluna não diferem estatisticamente entre si pelo teste de Scott Knott a 5\%.

Para os teores de água das sementes dos cinco lotes (Tabela 1) não se verifica variação elevada, o que é importante, pois a uniformidade do teor de água das sementes é essencial para a padronização das avaliações e resultados consistentes (Marcos Filho, 1999). Ainda de acordo com os dados da Tabela 1, verificou-se que as sementes dos lotes 1 e 2 apresentaram os maiores teores de água e não diferiram estatisticamente entre si. Por sua vez, o menor valor ocorreu nas sementes do lote 3 . Esses resultados contradizem com a afirmação de Carvalho \& Nakagawa (2000) quando mencionam que o elevado teor de água é uma das principais causas da perda do poder germinativo e do vigor das sementes.

Pelos dados da Tabela 2, constatou-se que apenas as sementes do lote 5 demonstraram porcentagem de germinação das sementes inferior aquelas dos demais lotes, os quais se encontravam com germinação acima de $96 \%$. Os resultados obtidos no teste de germinação corroboram com os mencionados por Marcos Filho (1999), uma vez que relatou que um dos objetivos fundamentais dos testes de vigor é detectar diferenças significativas na qualidade fisiológica de lotes de sementes com germinação semelhante, de forma a complementar as informações fornecidas pelo teste de germinação.

Resultados elevados do percentual de germinação, como foram obtidos no presente trabalho, não significa, necessariamente, que os lotes possuem alto vigor, uma 
vez que o teste de germinação é conduzido sob condições favoráveis de temperatura, luz, substrato e umidade, permitindo ao lote expressar o potencial máximo para produzir plântulas normais. Com isso, verifica-se a necessidade de aplicação de testes complementares. Entretanto, Fonseca et al. (2004) verificaram que o teste de germinação foi suficiente para separar em níveis de germinação os lotes de sementes de Ricinus communis L.

Os resultados obtidos no teste de germinação não estão de acordo com os obtidos para a emergência das plântulas no campo. Estima-se que em razão das condições adversas a que as sementes foram submetidas no teste de emergência em campo as diferenças de qualidade fisiológica de sementes entre os lotes testados tenham sido potencializadas. Assim, as diferenças não detectadas pelo teste de germinação, tornaram-se evidentes, como, pode ser observado entre os lotes 3 e 4 , os quais exibem a melhor condição fisiológica, obtendo os maiores percentuais de plântulas emergidas.

Para todos os lotes, a porcentagem de emergência das plântulas em campo foi inferior a de germinação, provavelmente em razão das condições ambientais do laboratório que normalmente são as mais adequadas para a espécie, o que não ocorrer em condições de campo, o que conduz a obtenção de menores valores em campo. Segundo Marcos Filho (1999), dentre os critérios de avaliação da confiabilidade de um determinado teste para avaliação da qualidade de sementes de diferentes lotes, a correlação dos resultados desse teste com os de emergência em campo é um dos mais adotados. Esse teste é considerado o melhor indicativo para inferir sobre o vigor de lotes de sementes, pois na sua execução devem ser utilizadas condições que simulem aquelas que as sementes estarão sujeitas por ocasião da semeadura em campo (Silveira et al., 2002).

Pelo teste de velocidade de emergência constatouse que as sementes do lote 1 e aquelas do lote 4 exibiram maior nível de vigor. No entanto, ao se avaliar a velocidade de germinação, observou-se que apenas as sementes do lote 1 e 5 mostraram-se com vigor inferior ao das demais
(Tabela 2). Pode ser que resultados discordantes dos referidos testes estejam associados ao princípio dos mesmos, pois são realizados em condições diferentes. Em Calendula officinalis L. foi possível a separação de lotes em níveis de vigor pelo teste de índice de velocidade de emergência (Silveira et al., 2002), bem como em sementes de Erytrhina velutina (Guedes et al., 2008).

Quanto ao comprimento da raiz principal das plântulas obtidas no teste de germinação, não se observou diferença significativa entre os lotes. No entanto, quando as plântulas vieram do campo, os lotes de sementes mais vigorosos foram 1 e 3 (Tabela 3 ). Mais uma vez constatase que apenas os dados de campo foram eficientes em separar os lotes em níveis de vigor.

O comprimento da parte aérea das plântulas do campo e do laboratório indicou as sementes dos lotes 3 como as mais vigorosas, enquanto o vigor das sementes dos demais lotes foi inferior (Tabela 3). Para sementes de soja, o teste de comprimento da raiz principal mostrou-se sensível para separação de lotes em níveis de vigor (Vanzolini et al., 2007), bem como para sementes de Erythrina velutina (Guedes et al., 2008).

Os resultados da massa seca das raízes e da parte aérea das plântulas do campo e laboratório são mostrados na Tabela 4. Com os dados de massa seca da raiz oriundas de plântulas do campo foi possível separar os lotes em níveis de vigor, sendo o lote 1 o que apresentou os maiores valores. Pode-se verificar que as sementes do lote 1 tinham maior peso de mil sementes (Tabela 1), portanto, isso pode ser a explicação para o melhor desempenho das sementes do lote 1 , no teste de massa seca da raiz principal, após germinação. Os lotes 1 e 5 obtiveram o maior conteúdo de massa seca da parte aérea das plântulas oriundas tanto do laboratório quanto do campo (Tabela 4).

Os testes realizados em campo (emergência, índice de velocidade de emergência e massa seca da parte aérea das plântulas em campo) podem ser utilizados como um indicativo para determinação do vigor de lotes de sementes de mulungu, pois apresentam uma resposta condizente com o teste de germinação.

Tabela 2 - Germinação, emergência de plântulas em campo, índice de velocidade de germinação - IVG e de emergência de plântulas em campo - IVE de sementes de Erytrhina velutina oriundas de cinco lotes.

\begin{tabular}{ccccc}
\hline Lotes & Germinação $(\%)$ & Emergência $(\%)$ & IVG & IVE \\
\hline $\mathrm{L}_{1}$ & $96 \mathrm{a}$ & $82 \mathrm{~b}$ & $6,84 \mathrm{a}$ & $4,39 \mathrm{a}$ \\
$\mathrm{L}_{2}$ & $99 \mathrm{a}$ & $76 \mathrm{~b}$ & $7,53 \mathrm{a}$ & $3,73 \mathrm{~b}$ \\
$\mathrm{~L}_{3}$ & $98 \mathrm{a}$ & $90 \mathrm{a}$ & $6,95 \mathrm{a}$ & $3,69 \mathrm{~b}$ \\
$\mathrm{~L}_{4}$ & $100 \mathrm{a}$ & $91 \mathrm{a}$ & $7,12 \mathrm{a}$ & $4,15 \mathrm{a}$ \\
$\mathrm{L}_{5}$ & $84 \mathrm{~b}$ & $75 \mathrm{~b}$ & $6,32 \mathrm{~b}$ & $3,56 \mathrm{~b}$ \\
\hline
\end{tabular}

Médias seguidas de mesma letra na coluna não diferem estatisticamente entre si pelo teste de Scott Knott a 5\%. 
Tabela 3 - Comprimento da raiz principal e da parte aérea de plântulas de Erytrhina velutina oriundas de sementes de cinco lotes.

\begin{tabular}{ccccc}
\hline \multirow{2}{*}{ Lotes } & \multicolumn{2}{c}{ Raiz principal } & \multicolumn{2}{c}{ Parte aérea } \\
\cline { 2 - 5 } & Laboratório & Campo & Laboratório & Campo \\
\hline $\mathrm{L}_{1}$ & $7,17 \mathrm{a}$ & $9,82 \mathrm{a}$ & $5,71 \mathrm{c}$ & $6,17 \mathrm{~b}$ \\
$\mathrm{~L}_{2}$ & $5,97 \mathrm{a}$ & $8,29 \mathrm{~b}$ & $6,53 \mathrm{~b}$ & $5,35 \mathrm{c}$ \\
$\mathrm{L}_{3}$ & $6,45 \mathrm{a}$ & $10,06 \mathrm{a}$ & $7,70 \mathrm{a}$ & $7,60 \mathrm{a}$ \\
$\mathrm{L}_{4}$ & $6,20 \mathrm{a}$ & $7,94 \mathrm{~b}$ & $6,29 \mathrm{~b}$ & $5,20 \mathrm{c}$ \\
$\mathrm{L}_{5}$ & $5,83 \mathrm{a}$ & $7,05 \mathrm{~b}$ & $6,85 \mathrm{~b}$ & $4,93 \mathrm{c}$ \\
\hline
\end{tabular}

Médias seguidas de mesma letra na coluna não diferem estatisticamente entre si pelo teste de Scott Knott a 5\%.

Tabela 4 - Massa seca da raiz principal e parte aérea de plântulas de Erytrhina velutina oriundas de sementes de cinco lotes.

\begin{tabular}{ccccc}
\hline \multirow{2}{*}{ Lotes } & \multicolumn{2}{c}{ Raiz principal } & \multicolumn{2}{c}{ Parte aérea } \\
\cline { 2 - 5 } & Laboratório & Campo & Laboratório & Campo \\
\hline $\mathrm{L}_{1}$ & $0,048 \mathrm{a}$ & $1,44 \mathrm{a}$ & $0,06 \mathrm{a}$ & $1,49 \mathrm{a}$ \\
$\mathrm{L}_{2}$ & $0,032 \mathrm{~b}$ & $0,57 \mathrm{~b}$ & $0,049 \mathrm{~b}$ & $0,75 \mathrm{c}$ \\
$\mathrm{L}_{3}$ & $0,030 \mathrm{~b}$ & $0,84 \mathrm{~b}$ & $0,050 \mathrm{~b}$ & $1,23 \mathrm{~b}$ \\
$\mathrm{~L}_{4}$ & $0,038 \mathrm{~b}$ & $0,93 \mathrm{~b}$ & $0,050 \mathrm{~b}$ & $1,12 \mathrm{~b}$ \\
$\mathrm{~L}_{5}$ & $0,032 \mathrm{~b}$ & $0,84 \mathrm{~b}$ & $0,06 \mathrm{a}$ & $1,49 \mathrm{a}$ \\
\hline
\end{tabular}

Médias seguidas de mesma letra na coluna não diferem estatisticamente entre si pelo teste de Scott-Knott a 5\%.

A análise geral dos dados obtidos permite verificar que nem todas as determinações realizadas foram eficientes para separar os lotes de sementes de $E$. velutina em níveis de vigor. Os lotes utilizados neste estudo eram heterogêneos em vários aspectos como origem, atributos genéticos e físicos, tornando mais complexa a comparação da sua qualidade. Carvalho \& Nakagawa (2000) enfatizaram que a origem da semente pode ter grande influência sobre seu comportamento, pelo menos, durante a germinação.

\section{CONCLUSÕES}

Os testes de campo (emergência de plântulas, índice de velocidade de emergência de plântulas e massa seca da parte aérea das plântulas) são eficientes na separação dos lotes de sementes de Erytrhina velutina Willd., em níveis de vigor.

\section{REFERÊNCIAS BIBLIOGRÁFICAS}

ASSOCIATION OF OFFICIAL SEED ANALYSTS. Seed vigour testing handbook. East Lasing, 1983. 88p.

(Contribution, 32).
BEWLEY, J.D.; BLACK, M. Seeds: physiology of development and germination. 2.ed. New York: Plenum, 1994. 445p.

BHERING, M.C.; DIAS, D.C.F.S.; TOKUHISA, D.;

DIAS, L.A.S. Avaliação do vigor de sementes de melão pelo teste de deterioração controlada. Revista

Brasileira de Sementes, Londrina, v.26, n.1, p.125-129, 2004.

BRASIL. Ministério da Agricultura e Reforma Agrária. Regras para análise de sementes. Brasília, DF: SNDA/ DNDV/CLAV, 1992. 365p.

CARNEIRO, J.G.A.; AGUIAR, I.B. Armazenamento de sementes. In: AGUIAR, I.B.; PIÑA-RODRIGUES, F.C.M.; FIGLIOLIA, M.B. (Eds.). Sementes florestais tropicais. Brasília, DF: ABRATES, 1993. p.333-350.

CARVALHO, N.M.; NAKAGAWA, J. Sementes: ciência, tecnologia e produção. 3.ed. Jaboticabal: FUNEP, 2000. $429 \mathrm{p}$. 
FONSECA, N.R.; MYCZKOWSKI, M.L.; PRIOR, M.; SÁ, R.O.; NAKAGAWA, J.; CAVARIANI, C.; ZANOTTO, M.D. Testes de avaliação da viabilidade e do vigor em sementes de mamona. In: CONGRESSO BRASILEIRO DE MAMONA: ENERGIAE SUSTENTABILIDADE, 1., 2004, Campina Grande, PB. Anais... Campina Grande, 2004.

GUEDES, R.S.; ALVES, E.U.; GONÇALVES, E.P.; VIANA, J.S.; LIMA, C.R. Avaliação do potencial fisiológico de sementes de Erythrina velutina Willd. pelo teste de comprimento de plântula. In: CONGRESSO DE PESQUISA E INOVAÇÃO DA REDE NORTE NORDESTE DE EDUCAÇÃOTECNOLÓGICA, 3., 2008, Fortaleza, CE. Resumos... Fortaleza, 2008. CDROM.

HAMPTON, J.G.; COOLBEAR, P. Potential versus actual seed performance can vigour testing provide an answer. Seed Science and Technology, Zürich, v.18, n.2, p.215228, 1990.

IRIGON, D.L.; MELLO, V.D.C. Análise de sementes. Brasília, DF: ABEAS, 1995. 88p.

KURIHARA, D.L.; IMAÑA-ENCINAS, J.; PAULA, J.E. Levantamento da arborização do campus da universidade de Brasília. Cerne, Lavras, v.11, n.2, p.127136, 2005.

LORENZI, H. Árvores brasileiras: manual de identificação e cultivo de plantas arbóreas do Brasil. Nova Odessa: Plantarum, 2002. 384p.
MAGUIRE, J.D. Speed of germination aid in selection and evaluation for seeding emergence and vigor. Crop Science, Madison, v.2, n.2, p.76-177, 1962.

MARCOS FILHO, J. Testes de vigor: importância e utilização. In: KRZYZANOWSKI, F.C.; VIEIRA, R.D.; FRANÇA NETO, J.B. (Eds.). Vigor de sementes: conceitos e testes. Londrina: ABRATES, 1999. p.1.1-1.21.

NAKAGAWA, J. Testes de vigor baseados na avaliação das plântulas. In: VIEIRA, R.D.; CARVALHO, N.M.

Testes de vigor em sementes. Jaboticabal: FUNEP, 1999. p.49-85.

SILVA, J.B.; VIEIRA, R.D. Avaliação do potencial fisiológico de sementes de beterraba. Revista Brasileira de Sementes, Brasília, v.28, n.2, p.128-134, 2006.

SILVEIRA, M.A.M.; VILLELA, F.A.; TILLMANN, M.Â.A. Comparação de métodos para avaliação da qualidade fisiológica em sementes de calêndula. Revista Brasileira de Sementes, Londrina, v.24, n.2, p.24-30, 2002.

TAIZ, L.; ZEIGER, E. Fisiologia vegetal. 3.ed. Porto Alegre: Artmed, 2006. 719p.

TORRES, S.B.; BRINGEL, J.M.M. Avaliação da qualidade sanitária e fisiológica de sementes de feijão macassar.

Caatinga, Mossoró, v.18, n.2, p.88-92, 2005.

VANZOLINI, S.; ARAKI, C.A.S.; SILVA, A.C.T.M.; NAKAGAWA, J. Teste de comprimento de plântula na avaliação da qualidade fisiológica de sementes de soja. Revista Brasileira de Sementes, Brasília, v.29, n.2, p.9096, 2007. 